\title{
USANDO REDES NEURAIS DIRETAS E REGRAS DE PRODUÇÃO NO CONTROLE DA CONCENTRAÇÃO DE HORMÔNIOS TIREOIDEANOS
}

\author{
L. G. A. Martins* \\ gustavo@facom.ufu.br
}

\author{
L. H. A. Monteiro ${ }^{\dagger}$ \\ luizm@mackenzie.br e luizmeusp.br
}

*Faculdade de Computação, Universidade Federal de Uberlândia

${ }^{\dagger}$ Escola de Engenharia, Pós-Graduação em Engenharia Elétrica, Universidade Presbiteriana Mackenzie e Departamento de Telecomunicações e Controle, Escola Politécnica, Universidade de São Paulo

\section{RESUMO}

O hipotireoidismo é uma patologia que ocorre no sistema de regulação dos hormônios tireoideanos, decorrente da deficiência na produção ou na ação desses hormônios. A maioria dos pacientes hipotireoideos deve fazer reposição hormonal durante toda a vida. Neste trabalho, desenvolve-se um modelo de controlador para o fornecimento de medicamentos aos pacientes com hipotireoidismo primário, devido à remoção total da glândula tireóide. Duas redes neurais diretas com múltiplas camadas predizem as concentrações dos hormônios TSH e $\mathrm{T}_{4}$ existentes no sangue. Essas previsões e as concentrações correntes são utilizadas como entrada para o controlador. Baseando-se em um conjunto de regras de produção, define-se qual a dosagem da droga que é necessária ser aplicada, no presente, a fim de manter as concentrações hormonais dentro de faixas de regularidade, no futuro. A atuação do controlador deve ser tal que mudanças bruscas não ocorram na evolução temporal das concentrações desses hormônios.

PALAVRAS-CHAVE: Hormônios tireoideanos, redes neurais, regras de produção.

\footnotetext{
Artigo submetido em 23/04/2006

1a. Revisão em 21/08/2006

2a. Revisão em 19/12/2006

Aceito sob recomendação do Editor Associado

Prof. Ivan Nunes Da Silva
}

\begin{abstract}
Hypothyroidism is a pathology that occurs in the system regulating the thyroid hormones and it is a consequence of insufficiency either in the production or in the action of these hormones. Most of the patients in hypothyroidism state must use hormonal replacement therapy for all their lives. In this work, a controller model for supplying therapeutic drugs to primary hypothyroidism patients, whose have been submitted to total removal of thyroid gland, is developed. Two multilayer perceptron neural networks predict the concentrations of the hormones TSH and $\mathrm{T}_{4}$ in the blood. These forecasts and the current concentrations are used as input to the controller. Based on a set of production rules, the controller defines the necessary drug dosage to maintain the concentrations inside regularity bands in the future. The performance of the controller must be such that abrupt changes do not occur on the time evolution of those hormones.
\end{abstract}

KEYWORDS: thyroid hormone, neural network, production rules.

\section{INTRODUÇÃO}

Um dos grandes desafios da medicina é o controle de doenças crônicas sem sintomas imediatos e claros, influenciadas por fatores externos, como dietas inadequadas, e por fatores de origem interna, como falha na produção e na secreção de hormônios. Para o controle dessas doenças, são necessários o monitoramento regular do quadro do paciente e a prescrição de tratamentos terapêuticos. Normalmente, esses 
tratamentos envolvem a administração oral ou intravenosa de medicamentos [Hacisalihzade, 1989].

Sob um enfoque de controle, esse tipo de doença resulta da falha de um ou mais elementos do sistema fisiológico de realimentação, o qual é responsável pela manutenção da homeostase do corpo humano [Carson e Deutsch, 1992]. Uma solução desse problema pode se dar através do desenvolvimento de um dispositivo capaz de avaliar o quadro do paciente, pela comparação entre as variáveis medidas e os valores desejados, e desencadear as ações de controle necessárias para a adequação dessas variáveis aos níveis considerados normais, restabelecendo o equilíbrio dinâmico do sistema.

Existem diversos estudos focados no desenvolvimento de mecanismos de regulação, que se dá pelo fornecimento de drogas para o tratamento das doenças. Nas últimas décadas, as abordagens utilizadas extrapolaram o escopo do controle clássico, adotando técnicas "inteligentes", com o intuito de aprimorar a flexibilidade do mecanismo em relação à dinâmica apresentada pelo processo. Dentre as várias abordagens utilizadas, as redes neurais artificiais vêm sendo largamente utilizadas nos processos de controle [Trajanoski e Wach, 1998], [Camps-Valls et alli, 2003]. Esse uso se justifica devido à sua capacidade de aprendizado e generalização.

Os sistemas endócrinos são fundamentais para o funcionamento do organismo humano. Dentre esses, destaca-se o sistema de regulação dos hormônios tireoideanos, que envolve a interação entre hipotálamo, hipófise e tireóide. Essa interação se dá através do transporte, pelo sangue, dos hormônios produzidos nesses órgãos. Entre as patologias que atingem esse sistema de regulação, destacam-se o hipertireoidismo e o hipotireoidismo.

O hipotireoidismo é resultante da deficiência de hormônios tireoideanos no organismo. Quando o defeito ocorre na própria tireóide, denomina-se hipotireoidismo primário [Carvalho, 2003], foco deste estudo. O tratamento é relativamente simples. A maioria dos pacientes hipotireoideos faz reposição hormonal, normalmente ingerindo $\mathrm{T}_{4}$ livre na forma de um sódio denominado levotiroxina.

Neste estudo, desenvolveu-se um mecanismo de controle para a dosagem de medicamentos em pacientes com hipotireoidismo primário. Esse controle baseia-se no emprego de duas redes neurais diretas com múltiplas camadas para a previsão das concentrações dos hormônios $\mathrm{TSH}$ e $\mathrm{T}_{4}$. A partir dessa previsão e da aferição das concentrações correntes, o dispositivo de controle utiliza-se de regras de produção para definir qual a quantidade da droga que deve ser administrada. Essa dosagem deve manter as concentrações dos hormônios dentro das respectivas faixas de regularidade, sem provocar mudanças bruscas na sua evolução temporal. Não se encontrou, na literatura, outro trabalho referente ao con- trole automático da concentração de hormônios tireoideanos. Para efeito de avaliação do mecanismo de controle proposto, comparou-se seu desempenho com o desempenho do método usado tradicionalmente pelos médicos, que adota uma dosagem fixa de medicamento.

Na seção 2, são descritos o sistema de regulação natural dos hormônios tireoideanos e suas síndromes principais. As redes neurais preditivas e o dispositivo de controle são apresentados na seção 3. A seção 4 contém os resultados obtidos nos experimentos numéricos realizados. As conclusões são descritas na seção 5. No apêndice A, são apresentados os modelos matemáticos propostos e empregados na geração dos dados para o treinamento das redes neurais e na simulação do mecanismo de controle.

\section{REGULAÇÃO DAS FUNÇÕES TIREOI- DEANAS}

O sistema endócrino de regulação do metabolismo celular funciona através do controle e do monitoramento sobre a produção e a secreção de hormônios pelo hipotálamo, pela hipófise e pela tireóide, como mostra a Figura 1. Além desses, o sangue e as células-alvo participam do processo, respectivamente, como meio de transporte e entidades "consumidoras" de tais hormônios.

Em seu funcionamento normal, o processo de regulação

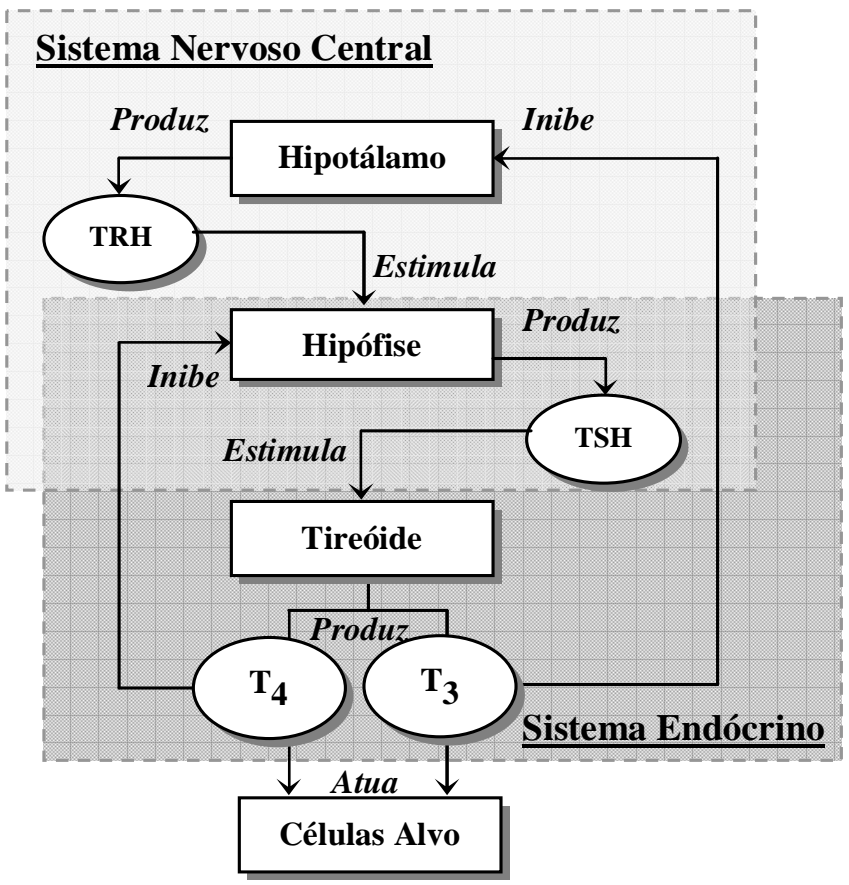

Figura 1: Sistema de regulação dos hormônios tireoideanos 
inicia-se com a produção e a liberação, pelo hipotálamo, do TRH (hormônio estimulante de tireotropina). Esse hormônio excita a glândula pituitária na síntese e secreção do TSH (hormônio estimulante da tireóide). O TSH, por sua vez, estimula a tireóide para a fabricação e a secreção dos hormônios tireoideanos $(\mathrm{TH})$, a saber, tiroxina $\left(\mathrm{T}_{4}\right)$ e triiodotironina $\left(\mathrm{T}_{3}\right)$ [Houssay et alli, 1972]. Isso garante um suprimento hormonal satisfatório para as células-alvo, que retiram do sangue a quantidade necessária para seu metabolismo. $\mathrm{O}$ nível de hormônios tireoideanos livres no sangue exerce um efeito de realimentação negativa sobre o suprimento de TSH, de modo que quanto maior a quantidade de TH no sangue, menor será a produção do TSH pela hipófise, e vice-versa. Outra possível conseqüência do incremento nos níveis de TH é a redução da densidade de receptores de TRH (TRHR) nas células da hipófise [Finke et alli, 1996]. Além disso, a função tireoideana é regulada, até certo ponto, por alguns fatores endógenos intratiroidais.

Entre as doenças que afetam o sistema de regulação dos hormônios tireoideanos, as principais são o hipertireoidismo e o hipotireoidismo [Robbins, 1969]. A primeira é um distúrbio que resulta em quantidades excessivas de hormônios tireoideanos circulantes, enquanto a segunda é resultante da deficiência dos mesmos.

O hipotireoidismo é uma síndrome clínica relativamente comum. No adulto, além da detecção clínica de alguns dos sintomas e sinais característicos, o diagnóstico é estabelecido pela aferição laboratorial das dosagens de $\mathrm{T}_{4}$ e TSH. Quando não tratado, o hipotireoidismo pode ocasionar sérios danos para a saúde, tais como cardiomegalia e retardo mental [Carvalho, 2003]. O tratamento padrão consiste na reposição hormonal através da administração oral diária do medicamento apropriado, proporcionando, assim, a quantidade supostamente adequada dos hormônios da tireóide. O controle do tratamento é realizado pelas concentrações de $\mathrm{T}_{4}$ livre e TSH no sangue, as quais devem ser mantidas sempre dentro do intervalo de normalidade (TSH de 0,4 a 2,0 $\mu \mathrm{UI} / \mathrm{ml}$ e $\mathrm{T}_{4}$ livre de 0,8 a $1,8 \mathrm{ng} / \mathrm{dl}^{1}$ ).

\section{MECANISMO DE CONTROLE}

O mecanismo desenvolvido é formado por duas redes neurais diretas e um controlador de dosagem, conforme ilustrado na Figura 2. Um modelo, baseado em equações diferenciais, representa a variação das concentrações hormonais do paciente a ser tratado. Com esse modelo, geram-se séries temporais relativas às concentrações de $\mathrm{T}_{4}$ livre e TSH, que são

\footnotetext{
${ }^{1}$ Foram considerados os valores de referência da faixa de normalidade do $\mathrm{T}_{4}$ livre e TSH obtidos em exames de sangue realizados, respectivamente, em 06/07/2003 e 26/04/2004, no laboratório do Hospital Sírio Libanês, por um dos autores, e em um trabalho publicado pela American Thyroid Association [Spencer et alli, 2002].
}

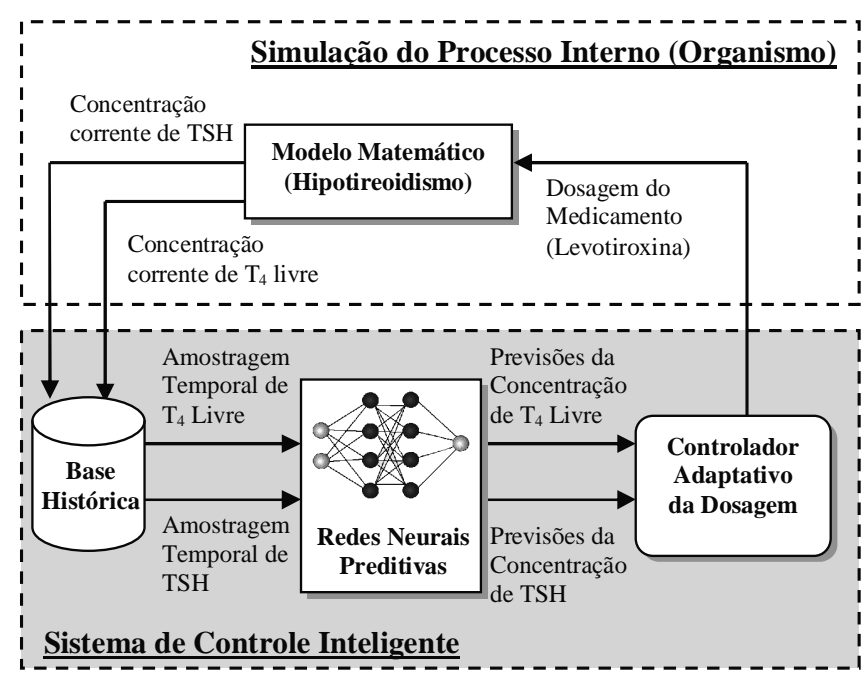

Figura 2: Mecanismo de controle

armazenadas numa base de dados. As duas redes neurais são treinadas com esses dados. Assim, a resposta do paciente à droga pode ser prevista por essas redes.

A partir das concentrações hormonais correntes e das previstas pelas redes neurais, o controlador utiliza um conjunto de regras de produção para determinar a quantidade da droga que deve ser administrada ao paciente hipotireoideo. Esperase que esse mecanismo seja capaz de prover a dosagem adequada, sem causar variações bruscas nas concentrações dos hormônios envolvidos no processo.

Todos os componentes do mecanismo de controle foram desenvolvidos usando o software Matlab $^{\circledR}$. A implementação das equações diferenciais foi realizada através do toolbox Simulink ${ }^{\complement}$, enquanto que o treinamento e a utilização das redes preditivas utilizaram funções pré-existentes do toolbox de redes neurais [Demuth e Beale, 1998]. A seguir, descrevese o dispositivo de controle utilizado nos experimentos numéricos. Os modelos para a dinâmica hormonal propostos e utilizados neste trabalho são apresentados no apêndice A.

\subsection{Predição}

Duas redes neurais artificiais são empregadas para a predição dos próximos valores das concentrações dos hormônios TSH e $\mathrm{T}_{4}$ livre, a partir de informações históricas das mesmas. Optou-se pela utilização de redes diretas com múltiplas camadas, também conhecidas por MLP (multilayer perceptrons), treinadas através do algoritmo error backpropagation. Essa escolha se deu pela farta bibliografia disponível [Argoud et alli, 2004], pelo seu desempenho satisfatório e pela facilidade de implementação. 
Nas redes MLP, os neurônios são organizados em camadas, sendo pelo menos uma camada escondida. A saída de um neurônio alimenta os neurônios da camada seguinte [Haykin, 1999].

Devido à dificuldade prática de se obter um conjunto relevante de dados reais sobre as variações das concentrações dos hormônios TSH e $\mathrm{T}_{4}$ livre, o conjunto de treinamento utilizado na aprendizagem das redes neurais foi obtido a partir de uma massa de dados gerada pelos nossos modelos matemáticos (ver apêndice A) dos indivíduos eutireoideo e hipotireoideo. Esse conjunto foi normalizado entre -1 e 1, e separado em três subgrupos. O primeiro engloba $50 \%$ da massa de dados e foi utilizado para o treinamento da rede. Os outros dois, com $25 \%$ cada, representam as bases de validação e de teste da rede.

Cada rede neural é composta por seis neurônios na camada de entrada e um único neurônio na camada de saída. Os neurônios de entrada representam as concentrações do respectivo hormônio no instante corrente $(t)$ e nas cinco medições antecessoras (entre $t-1$ e $t-5$ ). O neurônio de saída, por sua vez, representa o valor estimado da concentração do hormônio analisado para o próximo passo de tempo $(t+1)$. Cada passo de tempo corresponde a 12 horas na vida do paciente. Dessa forma, a predição das redes neurais baseia-se nas concentrações hormonais obtidas nos últimos três dias. As topologias das redes foram definidas a partir de inúmeros testes numéricos [Martins, 2004], sendo que a escolhida possui duas camadas escondidas. A rede neural de predição do TSH é composta por 24 neurônios na primeira camada intermediária e 12 na segunda, enquanto que a rede do $\mathrm{T}_{4}$ livre é formada por 12 e 6 neurônios, respectivamente. Todos os neurônios são dotados de uma mesma função de ativação sigmoidal do tipo tangente hiperbólica.

No treinamento das redes, os pesos sinápticos são ajustados pelo error backpropagation tradicional, a partir de uma inicialização aleatória. Foi utilizada uma taxa de aprendizagem de 0,1 e modo de treinamento incremental. Como critérios de parada, foram empregados o número máximo de 10.000 ciclos (cada ciclo refere-se à submissão de todo o conjunto de treinamento à rede), e margem de erro relativo de $1 \times 10^{-4}$. Para que a rede seja capaz de generalizar, evitou-se o overfitting, utilizando o método early stopping [Demuth e Beale, 1998].

\subsection{Controlador de dosagem}

O controle deve ser de tal forma que possibilite manter as concentrações do TSH e do $\mathrm{T}_{4}$ livre dentro de suas respectivas faixas de normalidade, sem provocar variações bruscas ao longo do tempo. Consideram-se, como variações bruscas, as oscilações superiores a 5\% em torno dos valores médios.
Para auxiliar na manutenção das concentrações do $\mathrm{T}_{4}$ livre e TSH dentro das respectivas faixas de normalidade, foi introduzido o conceito de faixa de segurança para cada uma das substâncias controladas. Os valores que delimitam essa faixa estão posicionados de modo proporcional às distâncias entre a concentração de equilíbrio (valor médio da faixa de normalidade) e os limites da faixa de normalidade, de modo que ela permaneça entre esses números. $\mathrm{O}$ cálculo desses valores limítrofes é feito pelas seguintes expressões:

$$
\begin{aligned}
& L S_{S}=\left[\left(L N_{S}-P E\right) \times 0,25\right]+P E \\
& L S_{I}=\left[\left(L N_{I}-P E\right) \times 0,25\right]+P E
\end{aligned}
$$

sendo $P E$ o valor da concentração de equilíbrio, $L S_{S}$ e $L S_{I}$ os valores do limite superior e do limite inferior da faixa de segurança, e $L N_{S}$ e $L N_{I}$ os valores do limite superior e do limite inferior da faixa de normalidade.

A estratégia de controle consiste, basicamente, na tomada de decisão de quanta droga liberar, a partir das concentrações correntes do $\mathrm{TSH}$ e do $\mathrm{T}_{4}$ livre e de suas tendências futuras, dadas pelas previsões das redes neurais. As concentrações das substâncias controladas no instante $t$ são utilizadas para determinar sua localização em relação às respectivas faixas de segurança, enquanto as previsões no instante $t+1$ são comparadas com esses valores, de modo a estimar a derivada da curva associada à evolução da concentração de cada hormônio. Dessa forma, espera-se que o controlador administre apenas a dosagem de manutenção previamente definida, se as concentrações dos hormônios estão dentro dos padrões desejados. Escolheu-se, como dosagem de manutenção, o valor de $Q$ no modelo do indivíduo hipotireoideo que resulte numa concentração de equilíbrio de $\mathrm{T}_{4}$ livre igual àquela obtida no modelo do indivíduo saudável $\left(\left[T_{4 L}\right] * \cong 1,08 \mathrm{ng} / \mathrm{dl}\right)$, ou seja, $Q * \cong 2 \mu \mathrm{g} / \mathrm{kg}$. Esse valor é satisfatório, dado que, no adulto, as doses de manutenção variam de 1,6 a 2,2 $\mu \mathrm{g}$ por kg do seu peso corporal. Quando as concentrações hormonais estiverem fora dos padrões desejados, o controlador aplica um percentual de ajuste sobre a dosagem de manutenção, com o objetivo de levar tais substâncias para aos patamares adequados.

A dosagem administrada não é totalmente absorvida pelo organismo [Zanini e Oga, 1994]. De fato, existe uma grande quantidade de variáveis envolvidas no processo de absorção e que, portanto, devem ser consideradas no cálculo da dosagem efetivamente absorvida $(Q(t))$. Entretanto, devido à dificuldade em modelar esse processo de absorção, optouse por assumir $Q(t)$ como sendo $80 \%$ da dosagem sugerida pelo controlador $\left(Q_{c}(t)\right)$, com um ruído branco gaussiano de $\pm 30 \%$. Como não se deseja a administração de doses superi- 
ores àquelas indicadas pelo controlador, os valores resultantes saturam em $100 \%$ de $Q_{c}(t)$.Como resultado, o organismo absorve de $50 \%$ a $100 \%$ da droga sugerida pelo controlador.

A quantidade da droga sugerida pelo controlador é obtida em função da dosagem de manutenção. Para a obtenção dessa quantidade, é utilizada uma média ponderada dos percentuais de ajuste $P_{T 4 L}$ e $P_{T S H}$. Nessa média, assume-se que o peso associado a $P_{T S H}$ é o dobro do associado a $P_{T 4 L}$, visando aumentar a influência do percentual de ajuste do TSH em relação ao do $\mathrm{T}_{4}$ livre. Dessa forma, o controlador adota uma estratégia inspirada no método tradicional, no qual, após a estabilização dos hormônios tireoideanos do paciente, o médico os controla apenas através da aferição da concentração do TSH. Por isso, assumimos que o peso associado ao TSH é maior do que aquele associado ao $\mathrm{T}_{4}$ livre. Assim, a dosagem sugerida $\left(Q_{c}\right)$ é dada por:

$$
Q_{c}=\frac{\left(P_{T 4 L}+2 P_{T S H}\right) \times Q *}{3}
$$

O percentual de ajuste de cada substância controlada $\left(P_{T 4 L}\right.$ e $\left.P_{T S H}\right)$ não pode ultrapassar $200 \%$ e é obtido através do conjunto de regras de produção descrito na Tabela 1. Cada regra é do tipo: $S E$ a concentração da substância está na região $R$ e apresenta a tendência $V, E N T A \tilde{O}$ o seu percentual de ajuste é de $X \%$ sobre a dosagem de manutenção.

Na definição dessas regras, consideramos inicialmente que os percentuais de ajuste devem ser de $100 \%$ da dosagem de manutenção $(Q *)$, quando as concentrações de $\mathrm{T}_{4}$ livre e TSH estiverem dentro das respectivas faixas de segurança e suas tendências, dadas pelas redes neurais, indicarem que aí permanecerão. A partir desses valores, definiram-se empiricamente os demais percentuais através do acréscimo ou decréscimo de $15 \%$, de acordo com a substância analisada e sua tendência, mantendo-se uma simetria na distribuição dos percentuais [Martins, 2004]. Nas situações extremas da tabela (abaixo do $L N_{I}$ com tendência de queda e acima do $L N_{S}$ com tendência de aumento), os valores foram ajustados com uma variação de $10 \%$ em relação às faixas mais próximas, para garantir que os valores de $P_{T S H}$ e $P_{T 4 L}$ fiquem dentro do intervalo de $0 \%$ a $200 \%$. Para $P_{T 4 L}=100 \%$ e $P_{T S H}$ $=100 \%$, o controlador administra apenas a dosagem de manutenção. Valores de $P_{T 4 L}$ e de $P_{T S H}$ diferentes de $100 \%$ indicam a necessidade de se ajustar essa dosagem: valores maiores que $100 \%$ mostram que se deve aumentar a quantidade da droga, valores menores revelam que a quantidade do medicamento fornecido deve ser diminuída.

\section{EXPERIMENTOS NUMÉRICOS}

Devido à dificuldade de realização de experimentos envolvendo pacientes reais, optou-se por fazer simulações numéricas, baseadas em nosso modelo matemático do paciente com
Tabela 1: Regras de produção utilizadas na definição dos percentuais de ajuste das substâncias controladas

\begin{tabular}{|c|c|c|c|}
\hline \multicolumn{2}{|c|}{ Análise da Concentração } & \multicolumn{2}{|c|}{$\begin{array}{l}\text { Perc. de } \\
\text { Ajuste }\end{array}$} \\
\hline Região & Tendência & $P_{T S H}$ & $P_{T 4 L}$ \\
\hline \multirow{3}{*}{ Acima do $L N_{S}$} & Aumento & $200 \%$ & $0 \%$ \\
\hline & Estabilidade & $190 \%$ & $10 \%$ \\
\hline & Queda & $175 \%$ & $25 \%$ \\
\hline \multirow{3}{*}{ Entre $L N_{S}$ e $L S_{S}$} & Aumento & $160 \%$ & $40 \%$ \\
\hline & Estabilidade & $145 \%$ & $55 \%$ \\
\hline & Queda & $130 \%$ & $70 \%$ \\
\hline \multirow{3}{*}{ Entre $L S_{S}$ e $P E$} & Aumento & $115 \%$ & $85 \%$ \\
\hline & Estabilidade & $100 \%$ & $100 \%$ \\
\hline & Queda & $100 \%$ & $100 \%$ \\
\hline \multirow{3}{*}{ Entre $P E$ e $L S_{I}$} & Aumento & $100 \%$ & $100 \%$ \\
\hline & Estabilidade & $100 \%$ & $100 \%$ \\
\hline & Queda & $85 \%$ & $115 \%$ \\
\hline \multirow{3}{*}{ Entre $L S_{I}$ e $N_{I}$} & Aumento & $70 \%$ & $130 \%$ \\
\hline & Estabilidade & $55 \%$ & $145 \%$ \\
\hline & Queda & $40 \%$ & $160 \%$ \\
\hline \multirow{3}{*}{ Abaixo do $L N_{I}$} & Aumento & $25 \%$ & $175 \%$ \\
\hline & Estabilidade & $10 \%$ & $190 \%$ \\
\hline & Queda & $0 \%$ & $200 \%$ \\
\hline
\end{tabular}

hipotireoidismo. Os resultados obtidos com o uso do controlador foram comparados com aqueles gerados através da simulação do tratamento tradicional, no qual uma dosagem de manutenção fixa do medicamento é administrada diariamente $(Q *=2 \mu \mathrm{g} / \mathrm{kg})$. Em ambos os casos, simulou-se um período de tratamento de 90 dias.

Os experimentos foram feitos em três situações distintas que correspondem aos quadros de pacientes hipotireoideos submetidos a tratamentos inadequados de dosagem irregular, subdosagem e superdosagem do medicamento. A primeiro situação visa representar pacientes que, apesar de apresentarem concentrações hormonais dentro da faixa de normalidade, possuem oscilações bruscas nos valores dessas concentrações, decorrentes da variação inadequada na quantidade do medicamento administrada. Nessas simulações, a dosagem de manutenção varia aleatoriamente entre 1,5 e 2,5 $\mu \mathrm{g} / \mathrm{kg}$. A segunda situação corresponde ao quadro de pacientes que, durante um período de tempo, suspenderam o tratamento; ou seja, estão sem receber a dosagem de manutenção do medicamento necessária para manter o equilíbrio no organismo. No terceiro caso, o paciente é submetido a 
uma dosagem diária excessiva do medicamento $(4 \mu \mathrm{g} / \mathrm{kg})$, resultando, assim, num quadro temporário de hipertireoidismo.

Os históricos das substâncias controladas foram gerados a partir de simulações com as equações diferenciais do paciente com hipotireoidismo, partindo-se dos valores de equilíbrio de cada substância e alterando-se os valores pertinentes à dosagem absorvida pelo organismo, de acordo com o tratamento pretendido. Para cada situação, foram criados aleatoriamente 100 cenários de amostragem, nos quais o período de tratamento varia de 5 a 8 dias.

Além da geração dos históricos, as equações diferenciais também foram empregadas nas simulações da resposta do paciente hipotireoideo aos tratamentos administrados. Nesse caso, as condições iniciais utilizadas para cada simulação foram obtidas a partir da última aferição de cada substância (no instante $t$ ), tendo, como ponto de partida, os históricos produzidos para cada situação; e da dosagem $Q(t)$ sugerida, após ter sido submetida ao fator de perturbação gerado naquele instante.

Para cada cenário, obtiveram-se as curvas das concentrações dos hormônios controlados (TSH e $\mathrm{T}_{4}$ livre) e das dosagens efetivamente absorvidas pelo organismo $(Q(t))$. A partir dessas curvas, calcularam-se as respectivas médias e desvios padrões das concentrações hormonais na fase de manutenção (após a estabilização), bem como a quantidade total de medicamento absorvido durante o tratamento e o tempo gasto para a regularização das concentrações hormonais dos pacientes. Visando padronizar o cálculo das médias, considerou-se que a regularização das curvas hormonais ocorria após 30 dias de tratamento (60 passos de simulação). Essa escolha foi inspirada no intervalo de tempo necessário para o medicamento atuar com aproximadamente $98 \%$ de sua capacidade total sobre o organismo e é, por isso, o período que os médicos geralmente esperam para verificar a resposta do organismo à dosagem administrada. Além disso, em nossas simulações, esse intervalo de tempo foi suficiente para garantir que, em todas as execuções, as curvas do TSH e do $\mathrm{T}_{4}$ livre já se encontrassem estabilizadas.

A Tabela 2 apresenta os resultados obtidos nas simulações com os três tipos de pacientes analisados: subdosagem, dosagem normal irregular e superdosagem. Para cada quadro, os resultados foram agrupados pelo método de controle empregado: dosagem fixa e adaptativa (com o uso do controlador). Nessa tabela, as colunas $\lambda$ e $\delta$ de cada concentração representam, respectivamente, a média dos valores médios de estabilização obtidos nas 100 simulações e a média dos seus desvios padrões. A coluna $\Theta$ refere-se ao maior desvio padrão médio obtido nas 100 simulações.

A primeira observação ao se analisar a tabela é que os resultados não variaram muito de um quadro para outro. Ou seja,
Tabela 2: Resultados obtidos em função do ambiente testado e da estratégia de controle adotada

\begin{tabular}{|c|c|c|c||c|c|c|}
\hline \multirow{2}{*}{$\begin{array}{c}\text { Método de } \\
\text { Controle }\end{array}$} & \multicolumn{3}{|c||}{$\begin{array}{c}\text { Concentração do } \\
T_{4} \text { Livre }\end{array}$} & \multicolumn{3}{c|}{$\begin{array}{c}\text { Concentração do } \\
T S H\end{array}$} \\
\cline { 2 - 7 } & $\lambda$ & $\delta(\%)$ & $\Theta(\%)$ & $\lambda$ & $\delta(\%)$ & $\Theta(\%)$ \\
\hline \multicolumn{6}{|c|}{ QUADRO DE SUBDOSAGEM } \\
\hline $\begin{array}{c}\text { Dosagem } \\
\text { fixa }\end{array}$ & 0,84 & 4,03 & 5,51 & 2,40 & 1,51 & 2,85 \\
\hline Adaptativo & 1,04 & 3,26 & 4,18 & 1,75 & 1,33 & 2,58 \\
\hline \multicolumn{7}{|c||}{ QUADRO DE DOSAGEM IRREGULAR } \\
\hline $\begin{array}{c}\text { Dosagem } \\
\text { fixa }\end{array}$ & 0,84 & 3,91 & 5,45 & 2,40 & 1,50 & 2,60 \\
\hline Adaptativo & 1,04 & 3,28 & 3,91 & 1,75 & 1,38 & 2,34 \\
\hline \multicolumn{2}{|c|}{ QUADRO DE SUPERDOSAGEM } \\
\hline $\begin{array}{c}\text { Dosagem } \\
\text { fixa }\end{array}$ & 0,84 & 3,96 & 5,20 & 2,40 & 1,54 & 2,90 \\
\hline Adaptativo & 1,04 & 3,28 & 3,81 & 1,75 & 1,43 & 2,51 \\
\hline
\end{tabular}

independentemente se o controle (dosagem fixa ou adaptativa) é realizado a partir de um quadro de subdosagem ou de dosagem normal irregular ou de superdosagem, comportamentos similares são obtidos. Isso se deve ao fato de que os resultados das concentrações apresentados na tabela foram obtidos a partir do trigésimo dia de tratamento, ou seja, após a estabilização no quadro do paciente.

Entretanto, nas três situações analisadas, observou-se que o uso do controlador resultou em valores médios $(\lambda)$ mais próximos aos pontos de equilíbrio das equações diferenciais. $\mathrm{O}$ método da dosagem fixa ocasionou oscilações bruscas nas concentrações do $\mathrm{T}_{4}$ livre, como pode ser observado nos resultados da coluna correspondente à oscilação máxima $(\Theta)$. Constatou-se, ainda, que o método de controle tradicional apresentou menor estabilidade relativa em relação à abordagem adaptativa; ou seja, durante a fase de manutenção, algumas das concentrações dos hormônios controlados saíram fora da faixa de normalidade. Tal conclusão pode ser tirada a partir dos resultados apresentados nas colunas referentes ao valor médio $(\lambda)$ e à oscilação média $(\delta)$. Além disso, observa-se que $100 \%$ dos cenários testados sem o uso do controlador apresentaram valores para a concentração do hormônio $\mathrm{T}_{4}$ livre abaixo do limite mínimo da faixa de normalidade.

Algumas observações adicionais, obtidas durante a realização dos experimentos, são apresentadas a seguir [Martins, 2004]. O tempo médio gasto para a normalização das concentrações dos hormônios controlados através do método de dosagem fixa é maior que aqueles obtidos através do controlador. No primeiro caso, o tempo médio para a normalização 
foi da ordem de 95 horas, enquanto que com o controlador demorou-se menos de 30 horas. Mas, na abordagem adaptativa, o paciente absorve em média $338 \mu \mathrm{g} / \mathrm{kg}$ durante todo o tratamento, enquanto que com a dosagem fixa essa absorção média é de $271 \mu \mathrm{g} / \mathrm{kg}$.

Na obtenção dos resultados apresentados na Tabela 2, foi empregado um ruído de $30 \%$ para a simulação das dosagens efetivamente absorvidas pelo organismo $(Q(t))$. Outros experimentos foram conduzidos com o objetivo de avaliar o comportamento do controlador e do método de dosagem fixa quando submetidos a diferentes níveis de ruído [Martins, 2004]. Avaliaram-se perturbações entre $10 \%$ e 50\%, e pode-se afirmar que os resultados do controlador são sempre superiores àqueles obtidos com a dosagem fixa, em relação aos valores médios obtidos e suas respectivas oscilações. Cabe ressaltar que quanto maior o nível de ruído, mais significativas são as melhorias obtidas com o uso do controlador em relação à dosagem fixa. Com controlador, para perturbações próximas a $\pm 50 \%$, verificou-se que os valores médios do $\mathrm{TSH}$ e do $\mathrm{T}_{4}$ livre permanecem dentro das respectivas faixas de normalidade, embora as oscilações nas concentrações hormonais excedam os 5\% desejados. Com dosagem fixa, perturbações da ordem de $30 \%$ já são suficientemente grandes e provocam oscilações bruscas (acima de 5\%) levando esporadicamente a concentração do $\mathrm{T}_{4}$ livre para fora de sua faixa de normalidade. Com $50 \%$ de ruído na dosagem fixa, aproximadamente $50 \%$ dos cenários avaliados resultaram em valores abaixo da normalidade.

\section{CONCLUSÕES}

O objetivo principal deste trabalho foi elaborar e avaliar um modelo de controlador capaz de tratar pacientes com hipotireoidismo primário, mantendo as concentrações dos hormônios $\mathrm{TSH}$ e $\mathrm{T}_{4}$ livre dentro das respectivas faixas de normalidade, sem provocar oscilações bruscas. Através dos experimentos, foi possível constatar que o controlador, de fato, conseguiu regularizar o quadro do paciente da forma desejada. Além disso, em nossas simulações, o tratamento administrado pelo controlador proporcionou, no geral, um comportamento mais adequado que nas simulações com a abordagem usualmente empregada de dosagem fixa.

De fato, o controlador se mostrou mais robusto a ruídos que a estratégia de dosagem fixa. Entretanto, a atuação do controlador sempre demanda uma quantidade maior de medicamento administrado. Em princípio, tal medicamento apenas repõe o hormônio que falta. Assim, usar uma maior ou menor quantidade não deveria comprometer o quadro clínico do paciente, dado que suas concentrações hormonais sejam mantidas dentro das faixas de normalidade pelo mecanismo de controle investigado.

\section{AGRADECIMENTOS}

Os autores agradecem a colaboração do Prof. J. G. S. C. M. Berlinck na concepção dos modelos de dinâmica hormonal apresentados no apêndice. LHAM agradece o apoio do CNPq.

\section{REFERÊNCIAS}

Argoud, F. I. M., Azevedo, F. M., Marino Neto, J. (2004). Sistema de Detecção Automática de Paroxismos Epileptogênicos em Sinais de Eletroencefalograma, Revista Brasileira de Controle \& Automação (SBA), Brasil, v. 15, n. 4, p. 467-475.

Brandt, M. E., Chen G. (2001). Feedback Control of a Biodynamical Model of HIV-1, IEEE Transactions on Biomedical Engineering, vol. 48, pp. 754-759.

Camps-Valls, G., Porta-Oltra, B., Soria-Olivas, E., MartínGuerrero, J. D., Serrano-López, A. J., Pérez-Ruixo, J. J., Jiménez-Torres, N. V. (2003). Prediction of Cyclosporine Dosage in Patients After Kidney Transplantation Using Neural Networks, IEEE Transactions on Biomedical Engineering, vol. 50, pp. 442-448.

Carson, E. R., Deutsch, T. (1992). A Spectrum of Approaches for Controlling Diabetes, IEEE Control Systems Magazine, vol. 12, pp. 25-31.

Carvalho, D. P. (2003). Hipotireoidismo: diagnóstico e princípios terapêuticos, thyroid Update, Sociedade Brasileira de Endocrinologia e Metabologia.

Demuth, H., Beale, M. (1998). Neural Network Toolbox For Use with MATLAB - User's Guide, The Math Works Inc., Massachusetts, USA.

Duro, R. J., Reyes J. S. (1999). Discrete-Time Backpropagation for Training Synaptic Delay-Based Artificial Neural Networks, IEEE Transactions on Neural Networks, vol. 10 , pp. 293-298.

Finke, R., Schleusener, H., Hierholzer, K. (1996). The thyroid gland: thyroid hormones, their origin and their mechanism of action, chapter 22. In: Comprehensive Human Physiology: From Cellular Mechanisms to Integration (Rainer Greger \& Uwe Windhorst, editors), Springer-Verlag, Berlim.

Fisher M. E. (1991). A Semiclosed-Loop Algorithm for the Control fo Blood Glucose Levels in Diabetics, IEEE Transactions on Biomedical Engineering, vol. 38, pp. 57-61.

Hacisalihzade, S. S. (1989). Control Engineering and Therapeutic Drug Delivery, IEEE Control Systems Magazine, vol. 9 , pp. 44-45. 
Haykin, S. (1999). Neural Networks - A Comprehensive Foundation, Prentice Hall, Upper Saddle River, NJUSA.

Houssay, B. A., Houssay, A. B., Hug, E., Leloir, L. F., Lewis, J. T., Soldati, L., Foglia, V. G., Fasciolo, J. C., Covian, M. R., Caldeyro-Barcia, R. (1972). Fisiologia Humana, $4^{a}$ edição, Libreria "El Ateneo" Editorial, Buenos Aires, Argentina.

Martins, L. G. A. (2004). Modelo de Controlador Inteligente para Regulagem de Hormônios Tireoideanos Baseado em Redes Neurais Preditivas, dissertação de mestrado, Universidade Presbiteriana Mackenzie, São Paulo.

Robbins, S. L. (1969). Patologia, Editora Guanabara Koogan, Rio de Janeiro, RJ-Brasil.

Spencer, C., Hollowell, J., Nicoloff, J., Braverman, L. (2002). Impact of TSH: TPOAb Relationships on Redefining the Serum TSH Normal Reference Range, 74th Annual Meeting of the American Thyroid Association, Los Angeles.

Trajanoski, Z., Wach, P. (1998). Neural Predictive Controller for Insulin Delivery Using the Subcutaneous Route, IEEE Trans. on Biomedical Engineering, vol. 45, pp. 1122-1134.

Zanini, A. C., Oga, S. (1994). Farmacologia Aplicada, Ed. Atheneu, São Paulo, SP-Brasil.

\section{APÊNDICE A - MODELO}

Elaboramos um modelo matemático, baseado em equações diferenciais, capaz de representar a dinâmica hormonal em questão. Com esse modelo, podem-se gerar dados a respeito das concentrações hormonais; contornando-se, assim, a dificuldade de se realizar experimentos envolvendo pacientes reais.

Dessa forma, foi possível gerar informações pertinentes às concentrações das substâncias envolvidas no processo de regulação hormonal da tireóide. O objetivo desse modelamento é produzir os dados a serem utilizados para o treinamento e validação das redes neurais, bem como para a simulação da dinâmica do paciente submetido ao tratamento sugerido pelo controlador.

Baseado nas interações entre hipotálamo, hipófise e tireóide, concebemos um modelo simplificado do processo normal de regulação dos hormônios tireoideanos (eutireoidismo), composto por equações que representam a variação temporal das concentrações de cada uma das principais substâncias envolvidas [Martins, 2004]. As equações diferenciais lineares que compõem nosso modelo são:

$$
\begin{gathered}
\frac{d[T R H]}{d t}=3-1,2[T R H]-0,8\left[T_{3} L\right]-0,7[T R H] \\
\frac{d[T S H]}{d t}=1,6[T R H]-1,2\left[T_{4} L\right]-0,5[T S H] \\
\frac{d\left[T_{G}\right]}{d t}=2[T S H]-0,8\left[T_{G}\right]-3,8\left[T_{G}\right]-0,02\left[T_{G}\right]
\end{gathered}
$$

$$
\frac{d\left[T_{3} L\right]}{d t}=0,38\left[T_{G}\right]-1,7\left[T_{3} L\right]+0,6\left[C_{3}\right]-0,002\left[T_{3} L\right]
$$

$$
\frac{d\left[T_{4} L\right]}{d t}=3,42\left[T_{G}\right]-1,8\left[T_{4} L\right]-0,004\left[T_{4} L\right]
$$

$$
\frac{d\left[C_{3}\right]}{d t}=1,7\left[T_{3} L\right]+1,4\left[C_{4}\right]-0,6\left[C_{3}\right]-0,7\left[C_{3}\right]
$$

$$
\frac{d\left[C_{4}\right]}{d t}=1,8\left[T_{4} L\right]-1,4\left[C_{4}\right]-0,8\left[C_{4}\right]
$$

sendo que:

- $P(t)=3$ representa o estímulo do sistema nervoso central na produção do TRH. Seu comportamento é influenciado pelas informações internas e externas ao organismo. Aqui, por simplicidade, assumiu-se um valor constante.

- As variáveis dependentes aparecem entre colchetes. São elas:

- [TRH]: concentração de TRH no sangue;

- [TSH]: concentração do TSH no sangue;

- $\left[T_{G}\right]:$ concentração de tireoglobulina iodada no colóide intratireoideano, ou seja, das moléculas que armazenam e transportam os $\mathrm{TH}$ dentro da tireóide;

- $\left[T_{4} L\right]$ : concentração de $\mathrm{T}_{4}$ na sua forma livre;

- $\left[T_{3} L\right]$ : concentração de $\mathrm{T}_{3}$ livre no sangue;

- $\left[C_{4}\right]$ : quantidade de $\mathrm{T}_{4}$ no interior das células;

- $\left[C_{3}\right]$ : quantidade intracelular de $\mathrm{T}_{3}$. 
- Cada termo das equações representa um tipo de interação entre as variáveis dependentes, como descrito a seguir:

- 1,2 [TRH] e $0,8\left[T_{G}\right]$ correspondem, respectivamente, ao processo de auto-inibição da síntese de TRH e da tireoglobulina;

- $0,8\left[T_{3} L\right]$ e $1,2\left[T_{4} L\right]$ representam o processo de inibição da síntese de TRH e TSH, a partir da quantidade sangüínea de $\mathrm{T}_{3}$ e $\mathrm{T}_{4}$ livres;

- 1,6[TRH] e $2[T S H]$ equivalem aos processos de estímulo do TRH à produção de TSH e desse sobre a síntese de TH;

- 3,8[ $\left.T_{G}\right]$ corresponde à clivagem da $\mathrm{T}_{G}$ existente na glândula tireóide em $\mathrm{TH}$, durante o processo de secreção desses hormônios;

- 0,38[T $\left.T_{G}\right]$ e $3,42\left[T_{G}\right]$ representam, respectivamente, os fatores de secreção dos hormônios $\mathrm{T}_{3}$ e $\mathrm{T}_{4}$, na sua forma livre, para o sangue. Em geral, a proporção desses termos em relação ao anterior é em torno de $10 \%$ e $90 \%$;

- $1,7\left[T_{3} L\right]$ e $1,8\left[T_{4} L\right]$ equivalem, respectivamente, aos fatores de absorção dos hormônios $\mathrm{T}_{3}$ e $\mathrm{T}_{4}$, na sua forma livre, pelas células-alvo;

- 1,4[C $\left.C_{4}\right]$ representa o fator de conversão do $\mathrm{T}_{4} \mathrm{em}$ $\mathrm{T}_{3}$ no interior das células-alvo;

- 0,6[C $\left.C_{3}\right]$ corresponde ao fator de liberação do hormônio $\mathrm{T}_{3}$ pelas células-alvo;

- Os demais termos equivalem aos fatores de decomposição das substâncias modeladas.

Modelos similares foram desenvolvidos para outros sistemas; por exemplo, no estudo da diabetes [Fisher, 1991], do HIV [Brandt e Chen, 2001] e da produção de sangue [Duro e Reyes, 1999].

Uma dificuldade encontrada durante o processo de identificação de valores para os parâmetros do sistema foi determinar a combinação adequada de modo a representar, de forma realista, as interações entre as subtâncias modeladas e, conseqüentemente, possibilitar que o ponto de equilíbrio dessas substâncias estivesse dentro das respectivas faixas de normalidade conhecidas. Os valores dos parâmetros foram inferidos a partir das concentrações de equilíbrio dos diversos hormônios e de informações coletadas na literatura [Martins, 2004].

A partir do nosso modelo para o indivíduo eutireoideo, foram realizadas alterações visando sua adaptação à dinâmica apresentada em pacientes com hipotireoidismo, após a remoção ou a destruição total da glândula tireóide. Nesse novo modelo, houve a remoção da equação (6) pertinente à tireoglobulina $\left(T_{G}\right)$ e dos parâmetros correspondentes à sua interação com $\mathrm{T}_{3}$ e $\mathrm{T}_{4}$ livres (nas equações (7) e (8), respectivamente), bem como a inclusão, na equação do $\mathrm{T}_{4}$ livre, de um novo termo para representar a absorção do hormônio sintético (levotiroxina) pelo organismo. A equação resultante é apresentada a seguir:

$$
\frac{d\left[T_{4} L\right]}{d t}=Q(t)-1,8\left[T_{4} L\right]-0,004\left[T_{4} L\right]
$$

sendo $Q(t)$ uma função que representa a quantidade do medicamento absorvida pelo organismo, em um determinado instante de tempo $t$. Sabe-se que nessa função são consideradas, entre outros fatores, a quantidade de medicamento administrado e a variação do grau de absorção conforme sua plenitude gástrica, uma vez que a droga é fornecida pela via oral [Zanini e Oga, 1994].

Vale ressaltar que o mecanismo de controle proposto neste trabalho independe do modelo matemático usado na geração dos dados empregados no treinamento da rede neural. Esse mecanismo é baseado em regras de produção e em predições de rede neural, que pode ser treinada ou com dados experimentais de indivíduos eutireoideos e pacientes com hipotireoidismo, ou então com dados gerados a partir de outros modelos. 